\title{
DMPL: An OpenMP DLL Debugging Interface
}

James Cownie, John DelSignore, Jr., Bronis R. de Supinski, and Karen Warren

This article was submitted to the Workshop on OpenMP

Applications and Tools 2003, Toronto, Canada, June 26-27, 2003

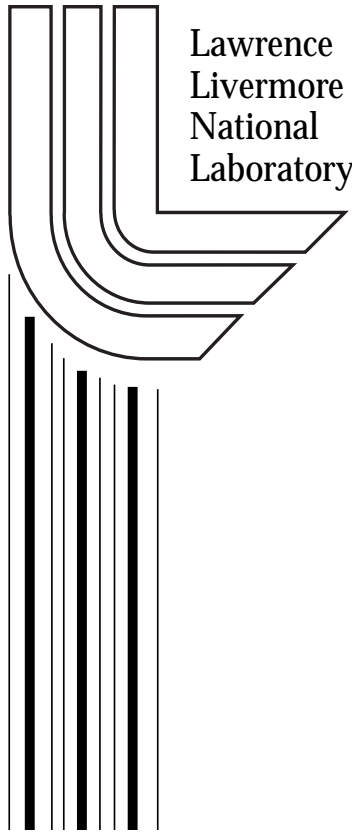

\section{April 8, 2003}




\section{DISCLAIMER}

This document was prepared as an account of work sponsored by an agency of the United States Government. Neither the United States Government nor the University of California nor any of their employees, makes any warranty, express or implied, or assumes any legal liability or responsibility for the accuracy, completeness, or usefulness of any information, apparatus, product, or process disclosed, or represents that its use would not infringe privately owned rights. Reference herein to any specific commercial product, process, or service by trade name, trademark, manufacturer, or otherwise, does not necessarily constitute or imply its endorsement, recommendation, or favoring by the United States Government or the University of California. The views and opinions of authors expressed herein do not necessarily state or reflect those of the United States Government or the University of California, and shall not be used for advertising or product endorsement purposes.

This is a preprint of a paper intended for publication in a journal or proceedings. Since changes may be made before publication, this preprint is made available with the understanding that it will not be cited or reproduced without the permission of the author. 


\title{
DMPL: An OpenMP DLL Debugging Interface
}

\author{
James Cownie ${ }^{1}$, John DelSignore, Jr. ${ }^{1}$, Bronis R. de Supinski ${ }^{2}$, and Karen Warren ${ }^{2}$ \\ ${ }^{1}$ Etnus, LLC, 24 Prime Parkway, \\ Natick, Massachusetts 01760 \\ \{jcownie, jdelsign\} eetnus.com \\ http: //www. etnus.com/Products/TotalView/index.html \\ ${ }^{2}$ Lawrence Livermore National Laboratory, P.O. Box 808, L-560, \\ Livermore, California 94551-0808* \\ \{bronis, kwarren\}@llnl.gov \\ http://www.linl.gov/icc/lc/DEG/TV.html
}

\begin{abstract}
OpenMP is a widely adopted standard for threading directives across compiler implementations. The standard is very successful since it provides application writers with a simple, portable programming model for introducing shared memory parallelism into their codes. However, the standards do not address key issues for supporting that programming model in development tools such as debuggers. In this paper, we present DMPL, an OpenMP debugger interface that can be implemented as a dynamically loaded library. DMPL is currently being considered by the OpenMP Tools Committee as a mechanism to bridge the development tool gap in the OpenMP standard.
\end{abstract}

\section{Introduction}

OpenMP is a widely used parallel programming model for shared-memory multiprocessor (SMP) architectures [1], [2]. The OpenMP organization initially focused on language design and run-time support. This focus has been successful - OpenMP now provides relative ease in writing codes that efficiently utilize SMP architectures.

While the initial focus has been successful, key areas for usability still need to be addressed. In particular, accurate information needed for debugging and analyzing performance of OpenMP applications can be difficult to obtain. Even when the information is provided, it is very difficult to present the information consistently with the semantics of the OpenMP programming model.

Recently, the OpenMP organization created a Tools Committee to address some of these difficulties. That committee is considering the POMP interface [3] as a standard method to instrument OpenMP applications and to gather performance data. However, the POMP interface does not address problems that arise when one attempts to debug an OpenMP code. In this document we propose DMPL (pronounced "dim-

\footnotetext{
* This work was partially performed under the auspices of the U.S. Department of Energy by University of California LLNLaboratory under contract W-7405-Eng-48. UCRL-JC-151670.
} 
ple"), an interface for OpenMP debugger support that can be implemented as a dynamically loaded library (DLL). We propose that each compiler vendor implement this library and provide it along with the OpenMP run-time library. Debuggers would then dynamically load this library to obtain the information needed for users to debug OpenMP codes.

The rest of this paper first explores key background issues for debugging OpenMP application codes. We then discuss why a DLL-based OpenMP debugger interface is the right solution. Next, we present an initial proposal for the definition of DMPL. We conclude with a discussion of why it is inappropriate to add the required debugger support to a performance instrumentation API and remaining open issues for OpenMP debugger support.

\section{Background}

\subsection{Multiple Compilers, User Levels}

Debugging features required for OpenMP codes are similar to those required for sequential code. Users want to work with the "source-level" code and to plant breakpoints within OpenMP regions. They want to step and otherwise control execution in those regions and to examine variables within them. However, the underlying threading provided by the OpenMP compiler and run-time complicates these goals. Should the debugger present outlined routines that the compiler creates so that OpenMP regions can be executed by multiple threads? Does the user expect the same view for shared, private, and thread private variables?

Some users understand how the OpenMP directives accomplish the desired parallelism; other users don't care about such details. Ideally, the debugger presents a serial code to the latter users while supporting a more detailed view that sophisticated users may desire. More realistically and practically, the debugger allows the user to see thread private variables and manipulate the threads individually. Regardless, a portable debugger must understand the output from various compilers (e.g., HP/Compaq, IBM, Intel-Guide, SGI and Sun) and present it to the user in a way that avoids involving the user in the underlying transformations.

\subsection{Compiler Transformations}

The OpenMP language specifications allow a variety of implementations. Portable debuggers must directly solve some possible implementation differences. However, a standard interface can assist the debugger in hiding many implementation details.

Depending on the compiler, the user's code may first be preprocessed and the resulting code actually compiled. The debugger deals with the preprocessed code. Since the user wants to debug the original code, the preprocessor must insert line number directives. The debugger must interpret the directives. Although line number direc- 
tives are already standardized, some OpenMP implementations that use preprocessing have not always included them.

The compiler uses threads to achieve the necessary parallelism. Various threading packages can be used: pthreads, sproc, and other proprietary threading packages. The debugger user must be able to asynchronously control these threads. This goal means that the underlying thread package must include support to synchronize thread execution, to single step threads in lockstep and for thread-specific breakpoints. Although the debugger must handle the underlying thread package, OpenMP implementers can help ensure that the package includes needed support for asynchronous thread control.

To achieve the desired parallelism, the compiler constructs outlined routines that the master thread calls and the worker threads execute. There may be multiple outlined routines for a single worksharing construct. Calls to the outlined routines become part of the stack traces. The stack frames from the routines become part of the calling stack frame. Ultimately, what looks like straight-line code to the user isn't. The debugger needs a standard method to recognize outlined routines and to associate them with worksharing constructs. Similarly, the debugger needs a standard mechanism to identify run-time library routines, for which the names vary for every compiler. Given these mechanisms, the debugger can then eliminate the calls from stack frames and support the appearance of straight-line code, if the user desires it.

Each compiler mangles the source code names differently when it makes up names for the outlined routines and their variables. The debugger must demangle a mangled name in order to present the user with a recognizable name. Further, the debugger may need to determine language meaning for the mangled name, such as whether a variable is shared or private. The debugger also must use the proper addressing mode for the variable, which can vary substantially between compilers, particularly for thread private data. The OpenMP implementation must provide the debugger with a standard mechanism for name demangling and this related information.

\section{DMPL Objective}

We propose DMPL, a dynamically loaded library interface that provides the debugger with information needed to support OpenMP applications. The TotalView parallel debugger [4] already uses this paradigm for debugging pthreads, MPI message queues [5], and UPC [6]. Similarly to run-time libraries, OpenMP compiler vendors would provide a DMPL library. The debugger, which already contains information about the run-time parallel environment, will load the DLL.

This paper defines the DMPL interface, which separates the OpenMP implementation from the debugger. This interface must provide a debugger with all the information that it needs to present the user with a view of his code both in terms of the original source and with additional thread details. The routines supplied by the vendor in the DLL can be linked dynamically in the debugger allowing callbacks to the debugger. The DLL is an OpenMP implementation-specific product and its implementation details are left to the OpenMP implementer. 


\section{DMPL Interface}

The proposed DLL is a two-way interface between the debugger and the DLL itself. When the debugger needs to display the value or address of a thread private object, it will make a call to a DLL function to extract the absolute address of the object. The DLL itself will make calls to the debugger to access information about the target process or thread. The DLL must not use global data internally because the debugger may be debugging independent processes simultaneously. Instead, it must associate data between calls with the specific object.

\subsection{DMPL Types}

DMPL includes several defined types in order to pass target architectural information to the debugger and to simplify interface function definitions. Other types provide function return codes, language values and codes for demangling information.

\begin{tabular}{|ll|}
\hline $\begin{array}{c}\text { typedef struct \{ } \\
\text { int short_size; }\end{array}$ & \\
int int_size; & \\
int long_size; & \\
int long_long_size; & /* sizeof (void * $) * /$ \\
int pointer_size; & \\
\} DMPL_target_type_sizes_t; & DMPL_taddr_t; \\
\hline typedef unsigned long long & DMPL_tword_t; \\
\hline typedef long long & DMPL_process_t; \\
\hline $\begin{array}{l}\text { typedef struct_DMPL_process_t } \\
\text { typedef struct_DMPL_thread_t } \\
\text { typedef struct_DMPL_type_t }\end{array}$ & DMPL_thread_t; \\
\hline
\end{tabular}

Fig. 1. Types for DMPL Target Architectural Information

Figure 1 shows that target architectural information includes the sizes of pointers and integer types and address values. Since DMPL_taddr_t is an unsigned long long, it allows for addresses on any architecture. Specifically, a 32-bit debugger should be able to debug a 64-bit target process.

\begin{tabular}{|ll|}
\hline enum \{ & Explanation \\
DMPL_ok=0, & Success \\
DMPL_tls_unallocated & No space allocated \\
DMPL_name_too_long, & Buffer too small \\
DMPL_name_changed, & Name wasn't demangled \\
DMPL_first_user_code = 100 & Allow more pre-defines \\
\hline$;$ & \\
\hline
\end{tabular}

Fig. 2. DMPL Result Codes

Figure 1 also includes opaque types for process, thread and type information. The debugger and the DLL will each determine what process/thread information it needs. The opaque types, which will be cast to concrete types in the debugger, preserve 
types across the DMPL interface. We use these undefined structures instead of void pointers in order to provide more compile-time checking at the cost of explicit casts in the library and support code.

\begin{tabular}{|lll|}
\hline enum \{ & & \\
DMPL_lang_unknown & $=$ & '' \\
DMPL_lang_c & $=$ & 'C', \\
DMPL_lang_cplus & $=$ & 'C', \\
DMPL_lang_f7 & $=$ & 'f'', \\
DMPL_lang_f9x & $=$ & 'F'' \\
\}; & & \\
\hline
\end{tabular}

Fig. 3. DMPL Language Codes

We have included three enumerated types in DMPL. In order to avoid potential issues with different compilers implementing enumerated types as different sized objects, we actually use int when they are used as a result or parameter type. Most DMPL functions return one of the result codes listed in Figure 2. Although both the DLL and the debugger will use values starting with DMPL_first_user_code, calling context will eliminate any confusion. Figure 3 shows the DMPL language values, which support providing the debugger with language-specific information from the OpenMP run-time library.

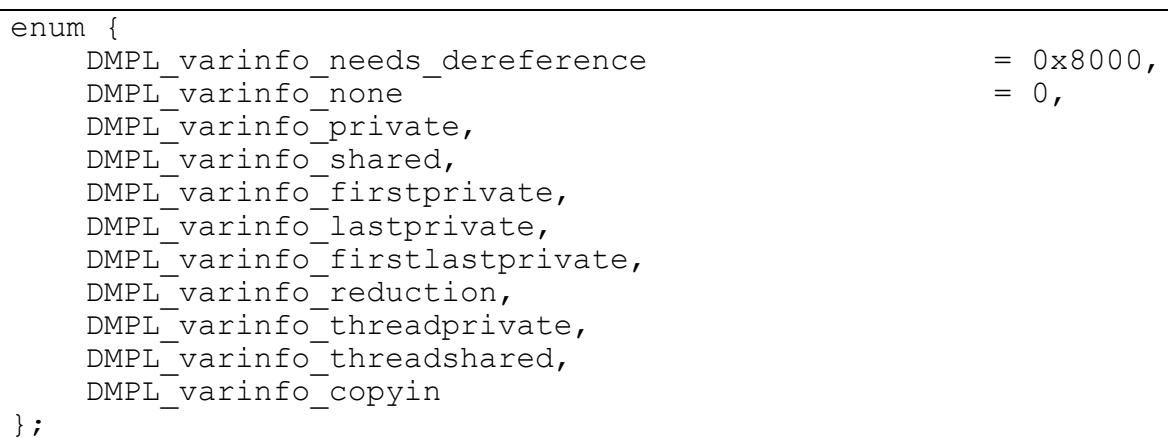

Fig. 4. DMPL Demangling Codes

The DLL uses DMPL demangling codes that are listed in Figure 4 to communicate scoping information to the debugger. DMPL_varinfo_needs dereference should be 'or-ed' into the appropriate scoping code in order to indicate that dereferencing is necessary to access the variable.

\subsection{DMPL Functions}

In order to access the information needed for OpenMP codes, the debugger loads the DMPL DLL. The debugger then calls the DMPL function DMPL_initialize, which performs steps necessary to initialize the DLL, including instantiation of a DMPL callback table that supports communication between the DLL and the debug- 


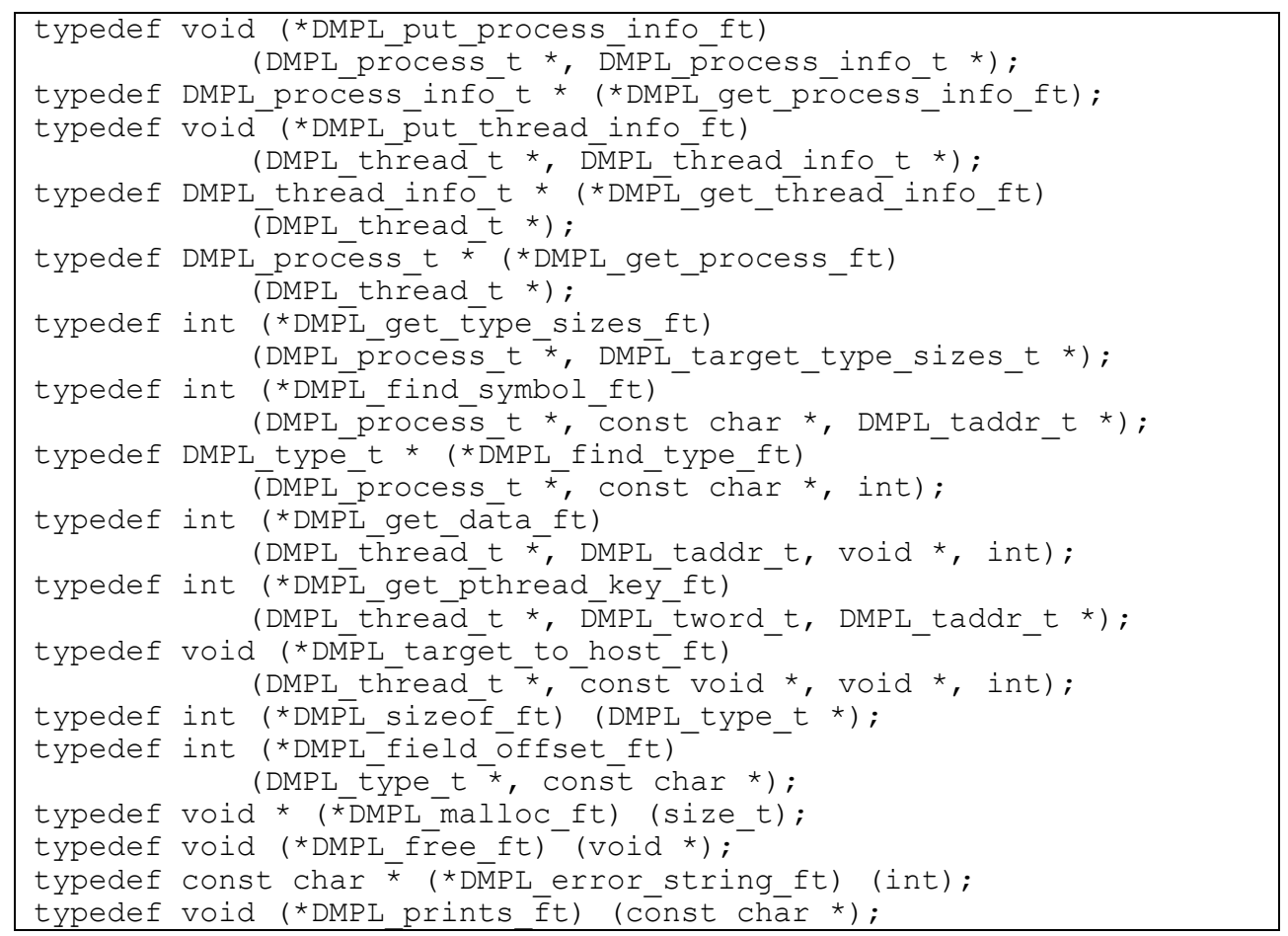

Fig. 6. DMPL Callback Function Type Definitions

The debugger uses two function types extensively to implement the type and data callback functions. A function of type DMPL_sizeof ft determines the size of a specific type. For structs and similar types, the DLL can find the field offset pointer of a member name of the type with a DMPL_get_field_offset_ft function.

The remaining callback functions provide important utility operations to the DLL. The DLL can allocate and free debugger memory with DMPL_malloc_fp and DMPL free fp. The DLL should not call malloc and free directly. The $\bar{D} L L$ can determine the error string associated with an error code by calling DMPL_error_string fp. DMPL_prints fp allows the DLL to print any messages. The $\bar{D} L L$ should not print messages directly.

\subsubsection{DMPL Debugger Functions}

The debugger accesses several functions in the DMPL interface. To display the value or address of the thread variable, the debugger will make calls to the DLL to get the absolute address of the object. The DLL also provides initialization, version information and the abilities to release process and thread information and to convert an error code to a string. The rest of this section describes the functions for debugger use, which are defined in Figure 7. 


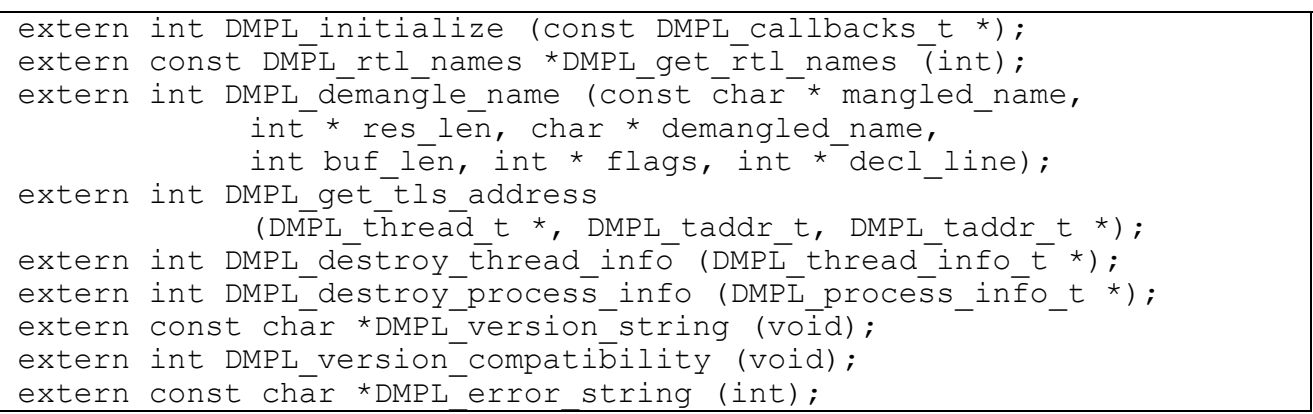

Fig. 7. DMPL Functions Accessed by the Debugger

The debugger calls DMPL_get_rtI_names in order to obtain the names of the run time library names invoked by a given OpenMP construct. The parameter of this function is a DMPL language code; as described previously, the parameter is passed as an int to avoid size conflicts. The return value is a pointer to a struct of two functions:

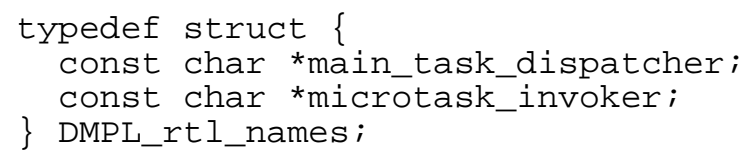

The main task dispatcher function is the outlined routine called by the main task to invoke the OpenMP parallel region or worksharing construct, which we refer to as a microtask. The second function invokes the actual microtask.

The DMPL interface provides the debugger with scoping functions to demangle mangled names and to locate the actual storage used for thread private variables. The res len parameter of the DMPL demangle name function allows the debugger to allocate more space for the result if it is too long for the initial demangled name buffer. Thread private data can be implemented in various ways: thread local storage system facilities, virtual-address-map page aliasing, and pthread key specific data. Thus, we provide DMPL_get_tls_address to obtain the actual address of a thread private object in this thread, given its apparent address in the process or thread.

The remaining functions in the DMPL interface that are used by the debugger provide important utility functions. The debugger releases any process or thread information associated by the DLL with a process (or thread) by calling DMPL destroy process info (or DMPL destroy thread info). The debugger ensures that the DL $\bar{L}$ provides the expected interface through the two versioning functions DMPL version string and DMPL_version_compatibility. Finally, DMPL_êrror_string converts error codes to strings for the debugger. 


\section{Discussion}

Although standards exist for OpenMP directives, there is currently no standard for the information that compilers or other tools require to present information consistently with the OpenMP programming model. Since compilers implement OpenMP directives differently, we propose that DMPL be adopted as a standard interface for providing debuggers and similar tools that information.

The POMP interface has already been proposed for providing information to performance tools. A legitimate question is whether performance tools and debuggers could be properly served by a unified information interface. However, the interfaces work in fundamentally different ways - the performance interface works within the application while the debugger interface is external. Compiler, run-time library and tool implementers have agreed during meetings of the OpenMP Tools Committee that they prefer two interfaces. The compiler writers and run-time system implementers have committed to providing two interfaces if the committee adopts them.

Even though the access mechanisms are sufficiently different to justify two standard information interfaces, there is significant intersection of the information needed for them. For example, DMPL includes functions to demangle names. Name demangling is also useful to performance tools. For this reason, we anticipate a set of lowlevel information standards or vendor supplied tools, such as a name demangler.

DMPL is a basic interface for the OpenMP debugging DLL. Implementations of this library with slightly different naming conventions are already available from IBM and Intel for its Guide compilers. OpenMP debugging on those platforms with current versions of TotalView demonstrates that DMPL provides significant support for the OpenMP programming model [4]. However, DMPL is an evolving interface and we recognize that additional functions and types may be needed. The Tools Committee will iterate on this specification and will eventually adopt a DLL-based debugger interface standard.

\section{References}

1. OpenMP Architecture Review Board: OpenMP Fortran Application Program Interface, Version 2.0. OpenMP Architecture Review Board (2000)

2. OpenMP Architecture Review Board: OpenMP C and C++ Application Program Interface, Version 2.0. OpenMP Architecture Review Board (2002)

3. Mohr, B., Malony, A.D., Hoppe, H.C., Schlimbach, F., Haab, G., Hoefflinger, J., Shah, S.: A Performance Monitoring Interface for OpenMP. In Proceedings of the Fourth European Workshop on OpenMP (EWOMP 2002). Rome (2002)

4. Etnus LLC: TotalView Reference Guide, Version 6.0. Etnus LLC (2002)

5. Cownie, J., Gropp, W.: A Standard Interface for Debugger Access to Message Queue Information in MPI. Sixth European PVM/MPI Users' Group Meeting. (1999)

6. Carlson, W.W., Draper, J.M., Culler, D.E., Yelick, K., Brooks, E., Warren, K.: Introduction to UPC and Language Specification. Technical Report CCS-TR-99-157, Institute for Defense Analysis, Center for Computer Sciences, Bowie, Maryland (1999) 